\title{
Waring's Problem in Finite Fields with Dickson Polynomials
}

\author{
Domingo Gomez and Arne Winterhof
}

\begin{abstract}
We study the problem of finding or estimating the smallest number of summands needed to express each element of a fixed finite field as sum of values of a Dickson polynomial. We study the existence problem and prove several bounds using results from additive number theory and bounds on additive character sums.
\end{abstract}

\section{Introduction}

Let $q=p^{r}$ be a power of a prime $p$ and denote by $\mathbb{F}_{q}$ the finite field of $q$ elements. We recall that the family of Dickson polynomials $D_{e}(X, \alpha) \in \mathbb{F}_{q}[X]$ is defined by the following recurrence relation

$$
D_{e}(X, \alpha)=X D_{e-1}(X, \alpha)-\alpha D_{e-2}(X, \alpha), \quad e=2,3, \ldots,
$$

with initial values

$$
D_{0}(X, \alpha)=2, \quad D_{1}(X, \alpha)=X,
$$

and $\alpha \in \mathbb{F}_{q}$. We refer to the monograph [8] for many useful properties and applications of Dickson polynomials.

Our aim is to study the following Waring problem with Dickson polynomials in finite fields.

We define $g_{\alpha}(e, q)$ as the smallest positive integer $s$ such that every $y \in \mathbb{F}_{q}$ can be expressed as

$$
y=D_{e}\left(u_{1}, \alpha\right)+\ldots+D_{e}\left(u_{s}, \alpha\right)
$$

with $u_{1}, \ldots, u_{s} \in \mathbb{F}_{q}$.

This problem has been studied for $\alpha=0$ by many authors, see $[\mathbf{1}, \mathbf{2}, \mathbf{5}, \mathbf{6}, \mathbf{1 1}$, $12,13]$ and references therein.

Here we focus on the case $\alpha=1$ but state the results for arbitrary $\alpha \neq 0$ if possible.

If $u=\mu+\alpha \mu^{-1} \in \mathbb{F}_{q}^{*}$ with $\mu \in \mathbb{F}_{q^{2}}$, the property

$$
D_{e}\left(\mu+\alpha \mu^{-1}, \alpha\right)=\mu^{e}+\alpha^{e} \mu^{-e},
$$

see [8], implies $D_{e}(u, \alpha)=D_{f}(u, \alpha)$ if $e \equiv f \bmod q^{2}-1$. Hence,

$$
g_{\alpha}(e, q)=g_{\alpha}\left(\operatorname{gcd}\left(e, q^{2}-1\right), q\right)
$$

1991 Mathematics Subject Classification. 11P05.

The first author is partially supported by the Project MTM2007-67088. He worked on the article during a pleasant research visit in Linz. 
and we may restrict ourselves to the case

$$
e \mid q^{2}-1
$$

In the case $r=1$ the number $g_{\alpha}(e, p)$ always exists. However, for $r>1$ it is possible that the value set of $D_{e}(X, \alpha)$ does not generate $\mathbb{F}_{q}$. For example, Equation (1.1) implies $g_{\alpha}\left(p^{2}-1, p\right)=p$ and $g_{\alpha}\left(q^{2}-1, q\right)$ does not exist for $r>1$.

We give necessary and sufficient conditions on the existence of $g_{1}(e, q)$ in Section 2. Sections 3 and 4 are devoted to bounds on $g_{\alpha}(e, q)$. We use results from additive number theory as well as bounds on additive character sums.

\section{Existence of $g_{1}(e, q)$}

In this section we characterize the pairs $(e, q)$ such that $g_{1}(e, q)$ exists.

TheOREM 2.1. Let $r=2^{u} v>1$ with an odd $v$. Then $g_{1}(e, q)$ exists if and only if one of the following two conditions holds

1. $\frac{q-1}{p^{d}-1}$ \e for all $d \mid r, d \neq r, \quad\left(p^{r / 2}-1\right)$ \e if $u \geq 1$,

$$
\text { and } \frac{q+1}{\operatorname{gcd}(2, p+1)} \text { \e if } v>1 \text {. }
$$

2. $\frac{q+1}{(2, p+1)}$ Xe and $\frac{q+1}{p^{d}+1}$ \e for all $d \mid r, d<r$, with $r / d$ odd.

In particular, $g_{1}(e, q)$ exists if $\operatorname{gcd}(e, q-1)<q^{1 / 2}-1$ or $\operatorname{gcd}(e, q+1)<\frac{3}{4} q^{2 / 3}$.

Proof. Put

$$
\mathbf{D}=\left\{D_{e}\left(u_{1}, 1\right)+\ldots+D_{e}\left(u_{s}, 1\right): u_{1}, \ldots, u_{s} \in \mathbb{F}_{q}, s \in \mathbb{N}\right\} .
$$

We have to characterize the conditions when $\mathbf{D}=\mathbb{F}_{q}$.

We consider the following vector spaces $\mathbf{A}$ and $\mathbf{B}$ over $\mathbb{F}_{p}$,

$$
\begin{aligned}
\mathbf{A}= & \left\{D_{e}\left(\mu_{1}+\mu_{1}^{-1}, 1\right)+\ldots+D_{e}\left(\mu_{s}+\mu_{s}^{-1}, 1\right): \mu_{1}, \ldots, \mu_{s} \in \mathbb{F}_{q}^{*}, s \in \mathbb{N}\right\}, \\
\mathbf{B}= & \left\{D_{e}\left(\mu_{1}+\mu_{1}^{-1}, 1\right)+\ldots+D_{e}\left(\mu_{s}+\mu_{s}^{-1}, 1\right): \mu_{1}^{q+1}=\ldots=\mu_{s}^{q+1}=1,\right. \\
& \left.\mu_{1}, \ldots, \mu_{s} \in \mathbb{F}_{q^{2}}^{*}, s \in \mathbb{N}\right\} .
\end{aligned}
$$

For $u \in \mathbb{F}_{q}^{*}$ the substitution $u=\mu+\mu^{-1}$ with $\mu \in \mathbb{F}_{q^{2}}^{*}$ implies either $\mu \in \mathbb{F}_{q}^{*}$ or $\mu^{q+1}=1$ since $u^{q}=\mu^{q}+\mu^{-q}=\mu+\mu^{-1}=u$. It is easy to see that

$$
\mathbf{D}=\mathbf{A}+\mathbf{B}=\{a+b: a \in \mathbf{A}, b \in \mathbf{B}\} \text {. }
$$

Since

$$
\begin{aligned}
& D_{e}\left(\mu_{1}+\mu_{1}^{-1}, 1\right) D_{e}\left(\mu_{2}+\mu_{2}^{-1}, 1\right)= \\
& D_{e}\left(\mu_{1} \mu_{2}+\left(\mu_{1} \mu_{2}\right)^{-1}, 1\right)+D_{e}\left(\mu_{1} \mu_{2}^{-1}+\mu_{1}^{-1} \mu_{2}, 1\right)
\end{aligned}
$$

by (1.1), we see that $\mathbf{A}$ and $\mathbf{B}$ are fields.

We note that $\mathbf{D}=\mathbb{F}_{q}$ implies $\mathbf{A}=\mathbb{F}_{q}$ or $\mathbf{B}=\mathbb{F}_{q}$.

The cardinality of $\mathbf{D}$ can be bounded by

$$
|\mathbf{A}+\mathbf{B}|<|\mathbf{A}||\mathbf{B}|
$$

since both fields contain $\mathbb{F}_{p}$. Using the fact that the cardinality of $|\mathbf{A}|=p^{d}$, $|\mathbf{B}|=p^{d^{\prime}}$, where $d, d^{\prime}$ are divisors of $r, q=p^{r}$, we get that $d=r$ or $d^{\prime}=r$. 
The problem has been reduced to prove in which cases

$$
\begin{aligned}
& \mathbf{A}_{1}=\left\{D_{e}\left(\mu+\mu^{-1}, 1\right): \mu \in \mathbb{F}_{q}^{*}\right\} \text { and } \\
& \mathbf{B}_{1}=\left\{D_{e}\left(\mu+\mu^{-1}, 1\right): \mu \in \mathbb{F}_{q^{2}}, \mu^{q+1}=1\right\}
\end{aligned}
$$

are both contained in a proper subfield.

If $\mathbf{A}_{1} \subset \mathbb{F}_{p^{d}}$ for some $d \mid r$ with $d \neq r$, we have

$$
\mu^{e}+\mu^{-e}=D_{e}\left(\mu+\mu^{-1}, 1\right)=D_{e}\left(\mu+\mu^{-1}, 1\right)^{p^{d}}=\mu^{e p^{d}}+\mu^{-e p^{d}}
$$

for any $\mu \in \mathbb{F}_{q}^{*}$, in particular, for a primitive element $\mu=g$ of $\mathbb{F}_{q}$. This implies $g^{e\left(p^{d}-1\right)}=1$ or $g^{e\left(p^{d}+1\right)}=1$ and thus

$$
e\left(p^{d}-1\right) \equiv 0 \bmod q-1 \quad \text { or } \quad e\left(p^{d}+1\right) \equiv 0 \bmod q-1 .
$$

If $\mathbf{B}_{1} \subset \mathbb{F}_{p^{d^{\prime}}}$ with $d^{\prime} \mid r$ and $d^{\prime} \neq r$ we get analogously

$$
e\left(p^{d^{\prime}}-1\right) \equiv 0 \bmod q+1 \quad \text { or } \quad e\left(p^{d^{\prime}}+1\right) \equiv 0 \bmod q+1
$$

The number $g_{1}(e, q)$ does not exist if and only if (2.2) and (2.3) both hold for some proper divisors $d$ and $d^{\prime}$ of $r$.

Finally, we simplify the conditions (2.2) and (2.3).

The first condition in $(2.2)$ is $\frac{q-1}{p^{d}-1} \mid e$.

If $r / d$ is odd, we have $\operatorname{gcd}\left(q-1, p^{d}+1\right)=\operatorname{gcd}\left(2, p^{d}+1\right)=\operatorname{gcd}(2, p+1)$ since $q-1 \equiv\left(p^{d}\right)^{r / d}-1 \equiv-2 \bmod p^{d}+1$ and thus the second condition in $(2.2)$ is $\frac{q+1}{\operatorname{gcd}(2, p+1)} \mid e$.

If $r$ is even and $d=r / 2$, the second condition in $(2.2)$ is $\left(p^{r / 2}-1\right) \mid e$.

If $r / d$ is even and $d<r / 2$, the second condition is covered by $\frac{q-1}{p^{2 d}-1} \mid e$.

Since $\operatorname{gcd}\left(p^{d^{\prime}}-1, q+1\right)=\operatorname{gcd}(2, p+1)$ the first condition in $(2.3)$ is $\frac{q+1}{\operatorname{gcd}(2, p+1)} \mid e$. If $r / d^{\prime}$ is odd, the second condition in $(2.3)$ is $\frac{q+1}{p^{d^{\prime}}+1} \mid e$.

If $r / d^{\prime}$ is even, the second condition in $(2.3)$ is $\frac{q+1}{\operatorname{gcd}(2, p+1)} \mid e$ which is already covered by the first condition in (2.3).

For arbitrary $\alpha$ a result of the same flavor cannot be obtained since $\mathbf{A}$ and $\mathbf{B}$ are not fields in general.

\section{Bounds based on addition theorems}

3.1. A consequence of the Cauchy-Davenport theorem. In this subsection we prove the following bound on $g_{\alpha}(e, p)$ based on the Cauchy-Davenport theorem.

THEOREM 3.1. We have

$$
g_{\alpha}(e, p) \leq 3 \min \{\operatorname{gcd}(e, p-1), \operatorname{gcd}(e, p+1)\}, \quad p \geq 3 .
$$

ProOF. For $s \geq 1$ put

$$
\mathbf{D}_{s}=\left\{D_{e}\left(u_{1}, \alpha\right)+\ldots+D_{e}\left(u_{s}, \alpha\right): u_{1}, \ldots, u_{s} \in \mathbb{F}_{p}\right\} .
$$

By the Cauchy-Davenport theorem we have

$$
\left|\mathbf{D}_{s}\right| \geq \min \left\{\left|\mathbf{D}_{s-1}\right|+\left|\mathbf{D}_{1}\right|-1, p\right\}, \quad s \geq 2,
$$

and get by induction

$$
\left|\mathbf{D}_{s}\right| \geq \min \left\{s\left(\left|\mathbf{D}_{1}\right|-1\right)+1, p\right\}, \quad s \geq 1 .
$$


By the formula of [3] for the cardinality of $\mathbf{D}_{1}$ we get

$$
\begin{aligned}
\left|\mathbf{D}_{1}\right| & \geq \frac{p-1}{2 \operatorname{gcd}(e, p-1)}+\frac{p+1}{2 \operatorname{gcd}(e, p+1)} \\
& \geq \max \left\{\frac{p-1}{2 \operatorname{gcd}(e, p-1)}, \frac{p+1}{2 \operatorname{gcd}(e, p+1)}\right\}+\frac{1}{2} .
\end{aligned}
$$

If $\operatorname{gcd}(e, p-1) \geq(p-1) / 2$, we get trivially $g_{\alpha}(e, p) \leq p \leq 3 \operatorname{gcd}(e, p-1)$. If $\operatorname{gcd}(e, p-1) \leq(p-1) / 3$, we get $\mathbf{D}_{s}=\mathbb{F}_{p}$ if

$$
s \geq 2 \operatorname{gcd}(e, p-1) \geq \frac{p-1}{(p-1) / 2 \operatorname{gcd}(e, p-1)-1 / 2} .
$$

If $\operatorname{gcd}(e, p+1) \geq(p+1) / 3$, we get $g_{\alpha}(e, p) \leq p \leq 3 \operatorname{gcd}(e, p+1)$.

If $\operatorname{gcd}(e, p+1) \leq(p+1) / 4$, we get $\mathbf{D}_{s}=\mathbb{F}_{p}$ if

$$
s \geq 3 \operatorname{gcd}(e, p+1) \geq \frac{p-1}{(p+1) / 2 \operatorname{gcd}(e, p+1)-1 / 2}
$$

and the result follows.

Note that the Cauchy-Davenport theorem is not valid in general for arbitrary finite fields.

For the case of prime fields and $\alpha=0$, sum-product techniques (see [5] and references therein) can be applied to derive very strong bounds on $g_{0}(e, p)$. It would be interesting to study this approach for $\alpha \neq 0$ as well.

3.2. Extension to arbitrary finite fields. In the case $\alpha=1$ we can reduce the problem of estimating $g_{1}(e, q)$ to the corresponding problem for prime fields.

Theorem 3.2. Let $q=p^{r}$. If $g_{1}(e, q)$ exists, then we have

$$
g_{1}(e, q) \leq 2 r \max \left\{g_{1}(d, p), g_{1}(f, p)\right\},
$$

where

with

$$
d=\frac{d_{1} d_{2}}{\operatorname{gcd}\left(d_{1}, d_{2}\right)}
$$

$$
d_{1}=\frac{p-1}{\operatorname{gcd}\left(\frac{(q-1)}{\operatorname{gcd}(e, q-1)}, p-1\right)} \quad \text { and } \quad d_{2}=\frac{p+1}{\operatorname{gcd}\left(\frac{(q-1)}{\operatorname{gcd}(e, q-1)}, p+1\right)}
$$

and

$$
f=\frac{f_{1} f_{2}}{\operatorname{gcd}\left(f_{1}, f_{2}\right)}
$$

with

$$
f_{1}=\frac{p-1}{\operatorname{gcd}\left(\frac{(q+1)}{\operatorname{gcd}(e, q+1)}, p-1\right)} \quad \text { and } \quad f_{2}=\frac{p+1}{\operatorname{gcd}\left(\frac{(q+1)}{\operatorname{gcd}(e, q+1)}, p+1\right)} .
$$

Proof. As in the proof of Theorem 2.1 we see that either $\mathbf{A}=\mathbb{F}_{q}$ or $\mathbf{B}=\mathbb{F}_{q}$. Thus, we can select $\left\{\beta_{1}, \ldots, \beta_{r}\right\}$ a basis of $\mathbb{F}_{q}$ over $\mathbb{F}_{p}$ that either $\left\{\beta_{1}, \ldots, \beta_{r}\right\} \subset \mathbf{A}_{1}$ or $\left\{\beta_{1}, \ldots, \beta_{r}\right\} \subset \mathbf{B}_{1}$.

Each element of $\mathbb{F}_{q}$ is a linear combination of $\left\{\beta_{1}, \ldots, \beta_{r}\right\}$ and Equation (2.1) states that the products of elements of $\mathbf{A}_{1}$ or $\mathbf{B}_{1}$ can be expressed as a sum of elements of $\mathbf{A}_{1}$ or $\mathbf{B}_{1}$, respectively. So we are going to investigate how many summands of elements of $\mathbf{A}_{1}$ and $\mathbf{B}_{1}$ are necessary to generate $\mathbb{F}_{p}$. 
First we suppose that $\left\{\beta_{1}, \ldots, \beta_{r}\right\} \subset \mathbf{A}_{1}$. For $\mu \in \mathbb{F}_{q}^{*}$ we have

$$
D_{e}\left(\mu+\mu^{-1}, 1\right)=\mu^{e}+\mu^{-e} \in \mathbb{F}_{p}
$$

if $\mu^{e} \in \mathbb{F}_{p}^{*}$ or $\mu^{e(p+1)}=1$ with $\mu \in \mathbb{F}_{p^{2}}^{*}$. The eth powers in $\mathbb{F}_{q}^{*}$ are the $(q-1) /(e, q-$ 1)th roots of unity and the elements of $\mathbb{F}_{p}^{*}$ are the $(p-1)$ th roots of unity in $\mathbb{F}_{q}^{*}$. Hence, the elements $\mu^{e} \in \mathbb{F}_{p}^{*}$ with $\mu \in \mathbb{F}_{q}^{*}$ are the $\left.((q-1) / \operatorname{gcd}(e, q-1), p-1)\right)$ th roots of unity or the $d_{1}=(p-1) /((q-1) / \operatorname{gcd}(e, q-1), p-1)$ th powers in $\mathbb{F}_{p}^{*}$. Similarly, we see that the eth powers $\mu^{e} \in \mathbb{F}_{q}^{*}$ with $\mu^{e(p+1)}=1$ are the $d_{2}=$ $(p+1) /((q-1) / \operatorname{gcd}(e, q-1), p+1)$ th powers of elements $\mu \in \mathbb{F}_{p}^{*}$ with $\mu^{p+1}=1$. Put $d=d_{1} d_{2} /\left(d_{1}, d_{2}\right)$. Hence, the values $D_{e}(u, 1) \in \mathbb{F}_{p}$ with $u \in \mathbb{F}_{q}$ coincide with the values $D_{d}(u, 1)$ with $u \in \mathbb{F}_{p}$. Now every element of $\mathbb{F}_{p}$ is sum of at most $g_{1}(d, p)$ summands. By (2.1) all elements $u \beta_{i}, u \in \mathbb{F}_{p}, i=1, \ldots, r$, are sums of $2 g_{1}(d, p)$ elements and we get the bound

$$
g_{1}(e, q) \leq 2 r g_{1}(d, p) .
$$

If we assume $\left\{\beta_{1}, \ldots, \beta_{r}\right\} \subset \mathbf{B}_{1}$, we obtain

$$
g_{1}(e, q) \leq 2 r g_{1}(f, p)
$$

analogously.

\section{Bounds derived by additive character sums}

Theorems 3.1 and 3.2 give general bounds for arbitrary finite fields which are up to a constant best possible since $g\left(p^{2}-1, p\right)=p$. However, these results can be improved using bounds on additive character sums if $\min \{\operatorname{gcd}(e, q-1), \operatorname{gcd}(e, q+1)\}$ is small. Note that in this case $g_{\alpha}(e, q)$ always exists.

Theorem 4.1. We have

$$
g_{\alpha}(e, q) \leq s \quad \text { if } \quad \operatorname{gcd}(e, q-1) \leq \frac{1}{8} q^{1 / 2-1 / 2(s-1)}, \quad s \geq 2 .
$$

For $\alpha=1$ we have additionally

$$
g_{1}(e, q) \leq s \quad \text { if } \quad \operatorname{gcd}(e, q+1) \leq \frac{1}{2} q^{1 / 2-1 / 2(s-1)}, \quad s \geq 2 .
$$

Proof. Without loss of generality we restrict ourselves to the cases when $s \geq 2$ and $e=\operatorname{gcd}(e, q-1)$ or $e=\operatorname{gcd}(e, q+1)$. First we consider the case $e=\operatorname{gcd}(e, q-1)$. In this case our technique works for all $\alpha$ whereas in the second case we need $\alpha=1$.

Let $\chi$ be a nontrivial additive character of $\mathbb{F}_{q}$. By

$$
\sum_{u \in \mathbb{F}_{q}} \chi(a u)= \begin{cases}0 & a \neq 0, \\ q & a=0,\end{cases}
$$

the number $N_{s}$ of solutions of the equation

$$
y=D_{e}\left(\mu_{1}+\alpha \mu_{1}^{-1}, \alpha\right)+\ldots+D_{e}\left(\mu_{s}+\alpha \mu_{s}^{-1}, \alpha\right), \quad \mu_{1}, \ldots, \mu_{s} \in \mathbb{F}_{q}^{*},
$$


is

$$
\begin{aligned}
N_{s} & =\frac{1}{q} \sum_{u \in \mathbb{F}_{q}} \sum_{\mu_{1}, \ldots, \mu_{s} \in \mathbb{F}_{q}^{*}} \chi\left(u\left(\sum_{i=1}^{s} D_{e}\left(\mu_{i}+\alpha \mu_{i}^{-1}, \alpha\right)-y\right)\right) \\
& =\frac{(q-1)^{s}}{q}+\frac{1}{q} \sum_{u \in \mathbb{F}_{q}^{*}} \sum_{\mu_{1}, \ldots, \mu_{s} \in \mathbb{F}_{q}^{*}} \chi\left(\sum_{i=1}^{s} u D_{e}\left(\mu_{i}+\alpha \mu_{i}^{-1}, \alpha\right)\right) \\
& =\frac{(q-1)^{s}}{q}+\frac{1}{q} \sum_{u \in \mathbb{F}_{q}^{*}}\left|\sum_{\mu \in \mathbb{F}_{q}^{*}} \chi\left(u D_{e}\left(\mu+\alpha \mu^{-1}, \alpha\right)\right)\right|^{s} .
\end{aligned}
$$

Since $e \mid q^{2}-1$ it is not divisible by $p$ and by [10, Lemma 2] we see that the rational function $X^{e}+\alpha^{e} X^{-e}$ is not of the form $A^{p}-A$. Hence, we can apply the character sum bound of Moreno and Moreno [9, Theorem 2] which implies

$$
\left(\max _{u \in \mathbb{F}_{q}^{*}}\left|\sum_{\mu \in \mathbb{F}_{q}^{*}} \chi\left(u D_{e}\left(\mu+\alpha \mu^{-1}, \alpha\right)\right)\right|\right)^{s-2} \leq\left(2 e q^{1 / 2}\right)^{s-2} .
$$

This implies that

$$
\left|N_{s}-\frac{(q-1)^{s}}{q}\right|<\frac{\left(2 e q^{1 / 2}\right)^{s-2}}{q} \sum_{u \in \mathbb{F}_{q}}\left|\sum_{\mu \in \mathbb{F}_{q}^{*}} \chi\left(u D_{e}\left(\mu+\alpha \mu^{-1}, \alpha\right)\right)\right|^{2}
$$

Expanding the inner sum, we get

$$
\sum_{\mu_{1}, \mu_{2} \in \mathbb{F}_{q}^{*}} \sum_{u \in \mathbb{F}_{q}} \chi\left(u\left(D_{e}\left(\mu_{1}+\alpha \mu_{1}^{-1}, \alpha\right)-D_{e}\left(\mu_{2}+\alpha \mu_{2}^{-1}, \alpha\right)\right)\right) .
$$

By (4.1), we get that the inner sum is zero, except if

$$
D_{e}\left(\mu_{1}+\alpha \mu_{1}^{-1}, \alpha\right)-D_{e}\left(\mu_{2}+\alpha \mu_{2}^{-1}, \alpha\right)=0 .
$$

For each $\mu_{1}$ there exist at most $2 e$ choices of $\mu_{2}$ such that this equation holds. So, this sum is at most $2 e q^{2}$. Substituting in (4.2), we get

$$
\left|N_{s}-\frac{(q-1)^{s}}{q}\right|<\left(2 e q^{1 / 2}\right)^{s-1} q^{1 / 2} .
$$

The number $N_{s}$ is positive for all $y \in \mathbb{F}_{q}$ if

$$
e \leq \frac{q^{1 / 2}}{8 q^{1 / 2(s-1)}}
$$

and thus $g_{\alpha}(e, q) \leq s$ under this condition.

Now we assume $e=\operatorname{gcd}(e, q+1)$ and $\alpha=1$, and denote by $N_{s}$ the number of solutions of

$$
y=D_{e}\left(\mu_{1}+\mu_{1}^{-1}, 1\right)+\ldots+D_{e}\left(\mu_{s}+\mu_{s}^{-1}, 1\right), \quad \mu_{1}^{q+1}=\ldots=\mu_{s}^{q+1}=1,
$$


where we need bounds on

$$
\max _{u \in \mathbb{F}_{q}^{*}}\left|\sum_{\substack{u \in \mathbb{F}_{q^{2}}^{*}, \mu^{q+1}=1}} \chi\left(u D_{e}\left(\mu+\mu^{-1}, 1\right)\right)\right| .
$$

Note that for $\mu$ with $\mu^{q+1}=\operatorname{Nm}_{\mathrm{q}^{2} / \mathrm{q}}(\mu)=1$ we have $D_{e}\left(\mu+\mu^{-1}, 1\right)=\mu^{e}+\mu^{-e}=$ $\mu^{e}+\mu^{e q}=\operatorname{Tr}_{\mathrm{q}^{2} / \mathrm{q}}\left(\mu^{e}\right)$.

Let $\psi$ be a multiplicative character of $\mathbb{F}_{q^{2}}$ of order $e$. Then we have

$$
\frac{1}{e} \sum_{j=0}^{e-1} \psi^{j}(\xi)= \begin{cases}1, & \xi=\mu^{e} \text { for some } \mu \in \mathbb{F}_{q^{2}}^{*}, \quad \xi \in \mathbb{F}_{q^{2}}^{*} . \\ 0, & \text { otherwise },\end{cases}
$$

Hence,

$$
\sum_{\substack{\mu \in \mathbb{F}_{q^{2}}^{*}, \mu^{q+1}=1}} \chi\left(u D_{e}\left(\mu+\mu^{-1}, 1\right)\right)=\sum_{j=0}^{e-1} \sum_{\substack{\xi \in \mathbb{F}_{q^{2}}^{*}, \operatorname{Nm}_{\mathrm{q}^{2} / \mathrm{q}}(\xi)=1}} \psi^{j}(\xi) \chi\left(\operatorname{Tr}_{\mathrm{q}^{2} / \mathrm{q}}(\xi)\right) .
$$

(Note that each $\xi$ which is an eth power equals $\mu^{e}$ for $e$ different $\mu$.) By [7, Theorem 2] the absolute value of the sum over $\xi$ can be bounded by $2 q^{1 / 2}$ and we get

$$
\left|N_{s}-\frac{(q+1)^{s}}{q}\right|<\left(2 e q^{1 / 2}\right)^{s-2} \sum_{u \in \mathbb{F}_{q}}\left|\sum_{\substack{\mu \in \mathbb{F}_{q^{2}}^{*}, \mu^{q+1}=1}} \chi\left(u D_{e}\left(\mu+\mu^{-1}, 1\right)\right)\right|^{2} .
$$

Following a similar reasoning as in the previous case, $g_{1}(e, q) \leq s$ if

$$
e \leq \frac{q^{1 / 2}}{2 q^{1 / 2(s-1)}}
$$

This finishes the proof.

Note that from [4, Theorem 10]

$$
g_{\alpha}(e, q) \leq s \text { if } \operatorname{gcd}(e, q-1)+\operatorname{gcd}(e, q+1) \leq \frac{q^{1 / 2}}{(q-1)^{1 / s}} .
$$

\section{References}

[1] Francis N. Castro, Ivelisse Rubio, Puhua Guan, and Raúl Figueroa. On systems of linear and diagonal equation of degree $p^{i}+1$ over finite fields of characteristic p. Finite Fields Appl., 14(3):648-657, 2008.

[2] Francis N. Castro, Ivelisse Rubio, and José M. Vega. Divisibility of exponential sums and solvability of certain equations over finite fields. Q. J. Math., 60(2):169-181, 2009.

[3] Wun Seng Chou, Javier Gomez-Calderon, and Gary L. Mullen. Value sets of Dickson polynomials over finite fields. Journal of Number Theory, 30:334-344, 1988.

[4] Wun Seng Chou, Gary L. Mullen, and Bertram Wassermann. On the number of solutions of equations of Dickson polynomials over finite fields. Taiwanese J. Math., 12(4):917-931, 2008.

[5] Todd Cochrane and Christopher Pinner. Sum-product estimates applied to Waring's problem mod p. Integers, 8:A46, 18, 2008.

[6] S. V. Konyagin. Estimates for Gaussian sums and Waring's problem modulo a prime. Trudy Mat. Inst. Steklov., 198:111-124, 1992. 
[7] Wen-Ching Winnie Li. Character sums over norm groups. Finite Fields Appl., 12(1):1-15, 2006.

[8] Rudolf Lidl, Gary L. Mullen, and Gerhard Turnwald. Dickson Polynomials. Longman, London-Harlow-Essex, 1993.

[9] Carlos Moreno and Oscar Moreno. Exponential sums and Goppa codes. Proceedings of the American Mathematical Monthly, 111:523-531, 1991.

[10] Harald Niederreiter and Arne Winterhof. Incomplete exponential sums over finite fields and their applications to new inversive pseudorandom number generators. Acta Arith., 93(4):387399, 2000.

[11] Christian van de Woestijne and Arne Winterhof. Exact solutions to waring's problem for finite fields. Acta Arith., to appear.

[12] Arne Winterhof. On Waring's problem in finite fields. Acta Arith., 87(2):171-177, 1998.

[13] Arne Winterhof. A note on Waring's problem in finite fields. Acta Arith., 96(4):365-368, 2001.

Faculty of Sciences, University of Cantabria, Avd. Los Castros, Santander, Spain. E-mail address: domingo.gomez@unican.es

Johann Radon Institute for Computational and Applied Mathematics, Austrian Academy of Sciences, Altenberger Strasse 69, A-4040 Linz, Austria.

E-mail address: arne.winterhof@oeaw.ac.at 